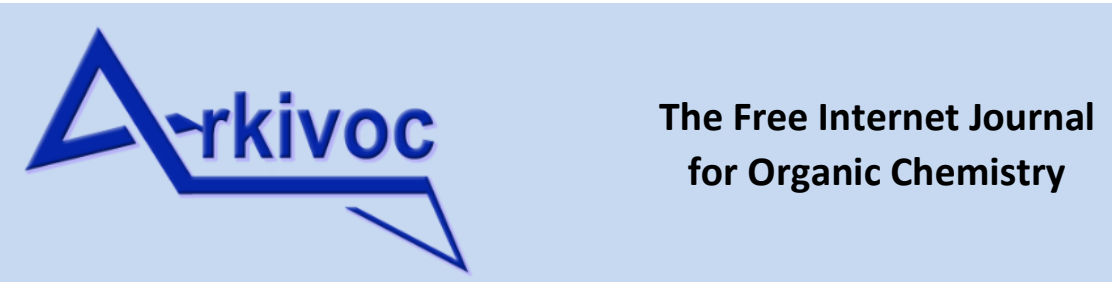

The Free Internet Journal

for Organic Chemistry

Paper

Archive for

Arkivoc 2021, part 0-0

Organic Chemistry

to be inserted by editorial office

\title{
Impact of the molecular structure of cationic binders on the rheological and adhesive properties of carboxymethyl cellulose-based coacervate hydrogels
}

\author{
William Megone, ${ }^{a, b}$ Pei Tang, ${ }^{a, b}$ Nima Roohpour ${ }^{c}$ and Julien E. Gautrot ${ }^{a, b}{ }^{*}$ \\ ${ }^{a}$ Institute of Bioengineering and ${ }^{b}$ School of Engineering and Materials Science, Queen Mary, University of \\ London, Mile End Road, London, E1 4NS, UK. \\ 'Consumer Healthcare R\&D, GlaxoSmithKline, St George's Avenue, Weybridge, Surrey, KT13 ODE, UK. \\ *Corresponding author.j.gautrot@qmul.ac.uk
}

This article is dedicated to Prof. Philip Hodge, for his advice as a mentor and inspiration as a scientist. Received mm-dd-yyyy

Accepted mm-dd-yyyy

Published on line mm-dd-yyyy

Dates to be inserted by editorial office

\section{Abstract}

In this work, we investigate the impact of organic oligo(cationic) binders on the rheological and adhesive properties of carboxymethyl cellulose. The behaviour of gels in the presence of organic oligo(cations) (ethylene imine-based), including block copolymers of poly(ethylene imine) and poly(ethyene glycol), is studied via oscillatory rheology and shear lap testing and compared to that of inorganic cations. We find that more polarisable inorganic cations $\left(\mathrm{Sr}^{2+}\right)$ and organic oligo(cations) display weaker rheological properties (storage shear moduli), as a result of the increased conformational collapse associated with corresponding complexes, compared to harder inorganic cations $\left(\mathrm{Ca}^{2+}\right)$. However, $\mathrm{Sr}^{2+}$ and block copolymers of oligo(ethylene imine)-based complex gels display increased adhesive strength, as a result of increased dissipative mechanisms.

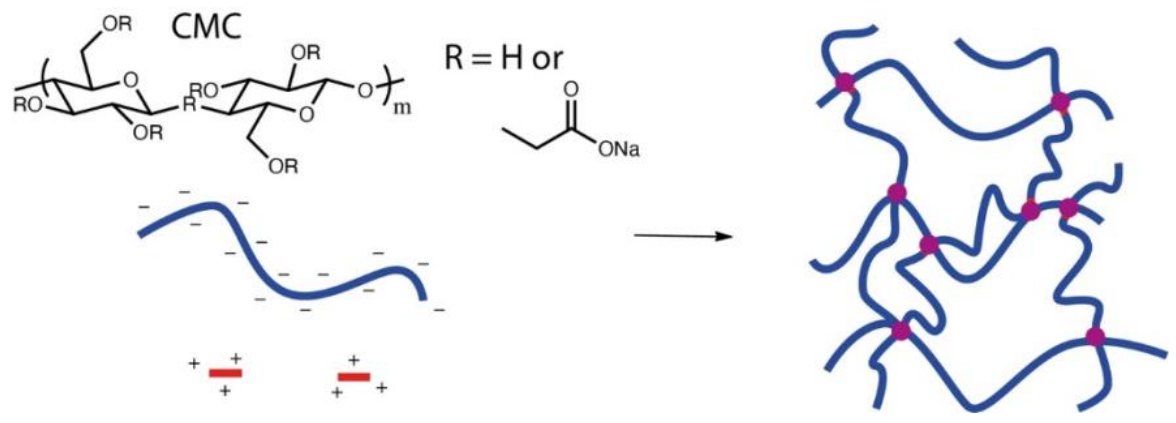

Keywords: Hydrogels, carboxymethyl cellulose, coacervate, polyelectrolytes. 


\section{Introduction}

Carboxymethyl cellulose (CMC) is a polysaccharide derived from cellulose by reaction with chloroacetic acid under alkaline conditions, displaying varying degrees of carboxylation (Figure 1). As cellulose is an abundant naturally occurring resource, $\mathrm{CMC}$ constitutes a very appealing material for the design of green biocompatible polymers. ${ }^{1-3} \mathrm{CMC}$ is currently used in a broad range of applications, from thickeners, binding agents, film formers, lubricants and stabilisers, particularly for food, to pharmaceutical and consumer care products. ${ }^{1-3} \mathrm{CMC}$ can be formulated into gels, either via physical or chemical crosslinking and has comparable physico-chemical properties to other polysaccharides such as gellan, alginate and xanthan..$^{1,4,5}$

CMC gels are also used extensively as denture adhesives. ${ }^{6-8}$ For such applications it is necessary that gels display suitable bulk mechanical and rheological properties, as well as high adhesive strength. In practice, denture adhesives must withstand complex loading and shear deformations during mastication. However, denture adhesives must also enable simple detachment of the denture without discomfort. This requires a balancing act, between sufficiently strong hold, bulk modulus and adhesion strength, and reversible bonding from denture and gum surfaces. Finally, the material should ensure a high level of comfort for the user, thought to be intimately correlated with the mechanics, microstructure and composition of corresponding gels. ${ }^{9-11}$

The solution properties (hydrodynamic diameter, viscosity) of CMC and the impact of its chemical structure and covalent crosslinking into hydrogel are well understood. ${ }^{1,2,12-14}$ lonic crosslinkers can also promote the formation of non-covalent networks and modulate rheological properties of corresponding solutions and gels. ${ }^{1}$ Similarly, other carboxylated polysaccharides, such as alginate, ${ }^{4,15}$ xanthan ${ }^{16}$ and gellan, ${ }^{17}$ can be crosslinked via the addition of cationic binders. In such systems, the negatively charged carboxylate groups on the polymer backbone can crosslink via electrostatic interaction with cations. Although such crosslinks are particularly strong in the case of calcium/alginate-based gels, cationic crosslinking of CMC gels is less efficient, presumably as the distribution of carboxylate moieties is random along the polymer backbone. ${ }^{18-20}$ Carboxylated polysaccharides crosslinked by multivalent cations typically lead to rigid local crosslinks, with some level of heterogeneity at the molecular scale. ${ }^{21,22}$ However, systems relying on softer crosslinked coacervate structures have also been proposed. For example, Hawker and co-workers have shown that poly(ethylene glycol) (PEG) based block copolymers, with oppositely charged end blocks form coacervates resulting in macroscopic hydrogels.

Similarly, the high degree of carboxylation typically achieved for CMC, and associated high charge density, enable the formation of hydrogel complexes in the presence of positively charged polyelectrolytes. ${ }^{2,23-25}$ For example, CMC forms homogenous polyelectrolyte polymer complex membranes and films when combined with chitosan ${ }^{24}$ or poly(vinyl amine). ${ }^{26}$ In these structures, CMC chains are proposed to be cross-linked primarily through a combination of hydrogen bonding and electrostatic interactions. ${ }^{24}$ Indeed, polysaccharide complexes occur typically as a result of primary electrostatic interactions between oppositely charged macromolecules, with associated increase in entropy from released counter-ions and the free energy gain arising from the formation of hydrogen bonding resulting in the stabilisation of the complex. A coacervate structure results from such assembly, which may lead to precipitation and full dehydration, depending on molecular architecture and environmental parameters (e.g. $\mathrm{pH}$ and ionic strength). ${ }^{21,27-31}$

More generally, the phenomenon of coacervation can be split into two types: complex coacervation and simple coacervation. A simple coacervate consists of a single macromolecule where polymer-polymer interactions are promoted over polymer-solvent interactions, for example in the presence of a dehydrating agent. A complex coacervate results from oppositely charged macromolecules complexing. ${ }^{29,}{ }^{30}$ Coacervation is predominantly an entropy driven process, enabled by the low translational entropy of the polyelectrolyte and gain in entropy associated with the release of counter-ions and the restructuring of water molecules solvating 
the macromolecules. ${ }^{30,31}$ As a results of such interactions, coacervates display weakly physically crosslinked yet hydrated structures that form distinct soft phases, often associated with phase separation, that are able to result in a network with altered viscoelastic properties (compared to the solution of separate initial polymers and compounds). The formation of coacervates is strongly affected by the ionic strength of the medium and associated reduction in the Debye length, resulting in the reduction in repulsive interactions between electrolyte blocks within the coacervate ${ }^{30-32}$ and modulating the entropic gain arising from counterion release..$^{30,31}$ Overall, $^{2}$ the stabilisation of coacervates depends on the ability to promote the formation of highly hydrated polyelectrolyte phases, rather than solid precipitation of dehydrated complexes. ${ }^{33,34}$ If polyelectrolyte interactions are too strong, complete dehydration and the restriction of polymer chain conformational freedom results in precipitation. In this respect, increasing the ionic strength can reduce the electrolyte strength, promoting coacervation. ${ }^{30}$ Similarly, the addition of ionic crosslinkers to charged hydrogels was shown to result in chain collapse and/or formation of micelles which reduce the interactions between polymer chains within the gel system. Therefore, a detailed understanding of the impact of the chemistry of cationic crosslinkers on CMC will help to shed light on the design of coacervate hydrogels and bioadhesives for biomedical and consumer care applications.

In this study, we explore how altering the chemical structure of cationic crosslinkers alters the mechanical performance of CMC hydrogels. The use of inorganic divalent cations, as well as multivalent organic cations and block copolymers with charged endcaps will be examined. The aim of this study is to correlate molecular structure with changes in shear modulus and adhesive strength of the CMC gels, as shown in Figure 1.
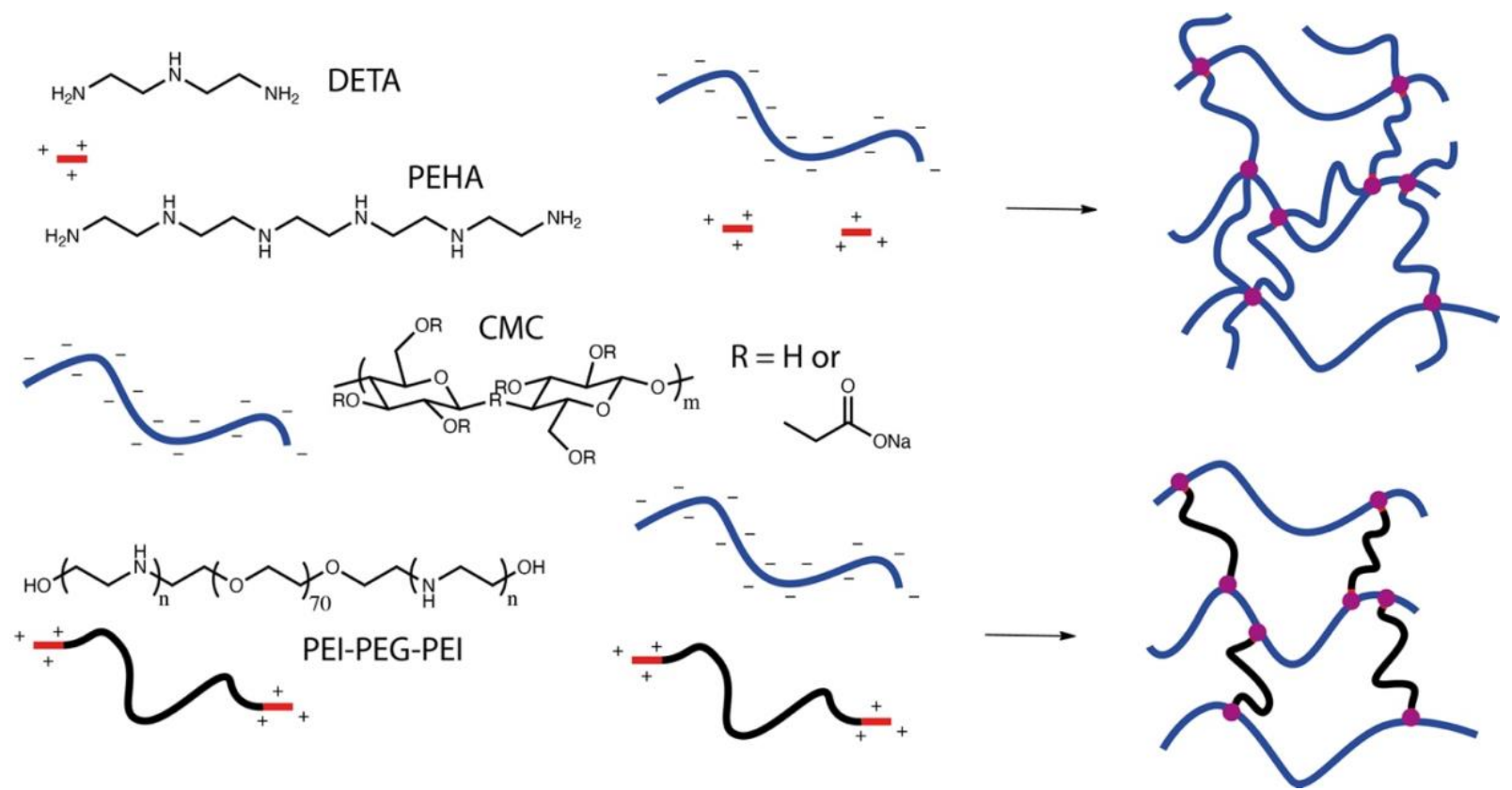

Figure 1. Chemical structure of CMC and organic electrolytes used in this work and schematic representation of the complexes they may form.

\section{Results and Discussion}

\section{Impact of Inorganic Electrolytes on PEG/CMC Gels}

We initially set out to examine the impact of combining inorganic divalent cations with PEG/CMC gels. It was thought that ionic crosslinking would alter the mechanical properties of the gel networks. The gels' mechanical properties were characterised by oscillatory rheology to investigate the impact of different cations. PEG/CMC 
gels were selected due to their applications as biomaterials in the field of consumer care technologies. The composition of the PEG/CMC gels used are shown in Table 1. Figure 2 presents the results obtained in these experiments.

Table 1. PEG/CMC and electrolyte compositions of hydrogels.

\begin{tabular}{|c|c|c|c|c|}
\hline $\begin{array}{l}\text { Sample } \\
\text { Name }\end{array}$ & $\begin{array}{l}\mathrm{CMC} \\
\text { Concentration } \\
\text { /wt\% }\end{array}$ & $\begin{array}{l}\text { PEG } \\
\text { Concentration } \\
\text { /wt\% }\end{array}$ & Electrolyte & $\begin{array}{l}\text { Molar Ratio } \\
\text { (CMC:Electrolyte) }\end{array}$ \\
\hline $\mathrm{NaCl}-5000$ & 3 & 1.5 & $\mathrm{NaCl}$ & 5000:1 \\
\hline $\mathrm{NaCl}-500$ & 3 & 1.5 & $\mathrm{NaCl}$ & $500: 1$ \\
\hline $\mathrm{NaCl}-50$ & 3 & 1.5 & $\mathrm{NaCl}$ & $50: 1$ \\
\hline $\mathrm{NaCl}-5$ & 3 & 1.5 & $\mathrm{NaCl}$ & $5: 1$ \\
\hline $\mathrm{CaCl}_{2}-5000$ & 3 & 1.5 & $\mathrm{CaCl}_{2}$ & 5000:1 \\
\hline $\mathrm{CaCl}_{2}-500$ & 3 & 1.5 & $\mathrm{CaCl}_{2}$ & $500: 1$ \\
\hline $\mathrm{CaCl}_{2}-50$ & 3 & 1.5 & $\mathrm{CaCl}_{2}$ & $50: 1$ \\
\hline $\mathrm{CaCl}_{2}-5$ & 3 & 1.5 & $\mathrm{CaCl}_{2}$ & $5: 1$ \\
\hline $\mathrm{SrCl}_{2}-5000$ & 3 & 1.5 & $\mathrm{SrCl}_{2}$ & 5000:1 \\
\hline $\mathrm{SrCl}_{2}-500$ & 3 & 1.5 & $\mathrm{SrCl}_{2}$ & $500: 1$ \\
\hline $\mathrm{SrCl}_{2}-50$ & 3 & 1.5 & $\mathrm{SrCl}_{2}$ & $50: 1$ \\
\hline $\mathrm{SrCl}_{2}-5$ & 3 & 1.5 & $\mathrm{SrCl}_{2}$ & $5: 1$ \\
\hline $\mathrm{HCl}-10000$ & 3 & 1.5 & $\mathrm{HCl}$ & 10000:1 \\
\hline HCl-1000 & 3 & 1.5 & $\mathrm{HCl}$ & 1000:1 \\
\hline $\mathrm{HCl}-100$ & 3 & 1.5 & $\mathrm{HCl}$ & $100: 1$ \\
\hline $\mathrm{HCl}-10$ & 3 & 1.5 & $\mathrm{HCl}$ & $10: 1$ \\
\hline $\mathrm{HCl}-1$ & 3 & 1.5 & $\mathrm{HCl}$ & $1: 1$ \\
\hline
\end{tabular}



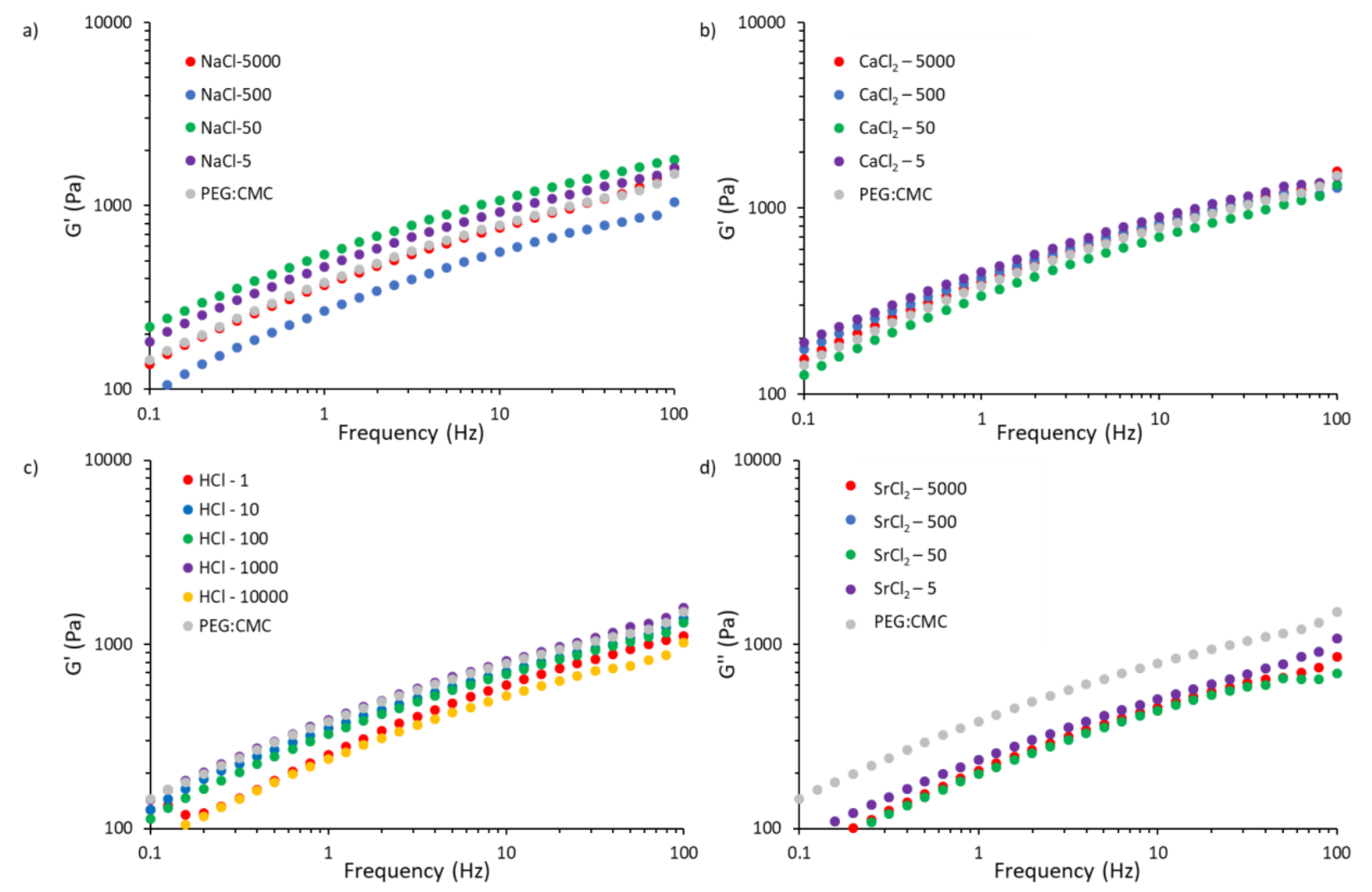

Figure 2. Representative frequency sweeps (shear storage modulus as a function of frequency) carried out via oscillatory rheology on PEG/CMC gels with 3 wt\% CMC and 1.5 wt\% PEG and varying (a) $\mathrm{NaCl}$, (b) $\mathrm{CaCl}_{2}$, (c) $\mathrm{HCl}$ and (d) $\mathrm{SrCl}_{2}$, at concentrations indicated in Table 1. PEG:CMC corresponds to data of gels without added electrolytes. Frequency sweeps were carried out at room temperature with an oscillating displacement of $10^{-4}$ rad from $0.1-100 \mathrm{~Hz}$.

All PEG/CMC gels, with and without inorganic electrolytes, display comparable frequency-dependent shear moduli profiles (Figure 2). This is expected from physical gels with large water content, leading to viscoelastic and poro-viscoelastic mechanical properties. $5,36,37$ The observation of frequency dependent moduli is common to physical gels as the network mechanics stems from a combination of physical crosslinks and polymer chain entanglement which, when strained at high frequency will lock together and cannot relax sufficiently fast, leading to stiffening of the network. In contrast, when strained at lower frequencies chains can slide past one another and physical crosslinks can dynamically rearrange, resulting in weaker networks. Such behaviour was previously reported for a broad range of hydrogels, including CMC gels ${ }^{5}$ and chitosan/poly(vinyl alcohol) gels. ${ }^{36}$

However, these experiments clearly indicated that the shear modulus of the corresponding PEG/CMC gels is not sensitive to the concentration of the inorganic electrolyte crosslinkers selected. While this was expected from the addition of $\mathrm{NaCl}^{16}$ as this is a monovalent electrolyte, it was expected that divalent cations would induce the strengthening of networks, as in the case of alginate ${ }^{18-20,38}$ and gellan. ${ }^{17,39}$ This implies that the ionic interactions between the negative pendant charges on the CMC backbone and the divalent salts are not resulting in efficient crosslinking between chains. Alternatively, it may also be proposed that local sample crosslinking results in some heterogeneity, and the formation of isolated "hard" hydrogel pockets that do not contribute to strengthening of the mechanical properties of the corresponding hydrogels. The dual PEG network may also be influencing the efficacy of the ionic crosslinking via the chelation of cations by PEG chains, hence reducing the formation of cationic bridges between $\mathrm{CMC}$ chains. ${ }^{40} \mathrm{CMC}$ with a high degree of substitution (> 
1.2) has also been shown to aggregate in dual polymer networks as a result of hydrogen bonding, hence the cationic electrolyte solution may be contributing to the formation of intramolecular complexes rather than forming crosslinks. ${ }^{26}$

The impact of PEG on CMC hydrogel rheology was also characterised, with the mechanics of native PEG gels also investigated (Figure 3a). Native PEG gels with wt\% from $0.13 \%$ to $6.25 \%$ were studied and PEG/CMC gels across the same range of PEG concentrations were combined with 12.5 wt\% CMC to investigate the impact of PEG on CMC hydrogel networks. The influence of Pluronic ${ }^{\circledR}$ (a non-ionic surfactant displaying a triblock copolymer structure based on ethylene oxide and propylene oxide, PEO-PPO-PEO) on the mechanics of CMC gels was also investigated to compare with the results obtained for PEG.

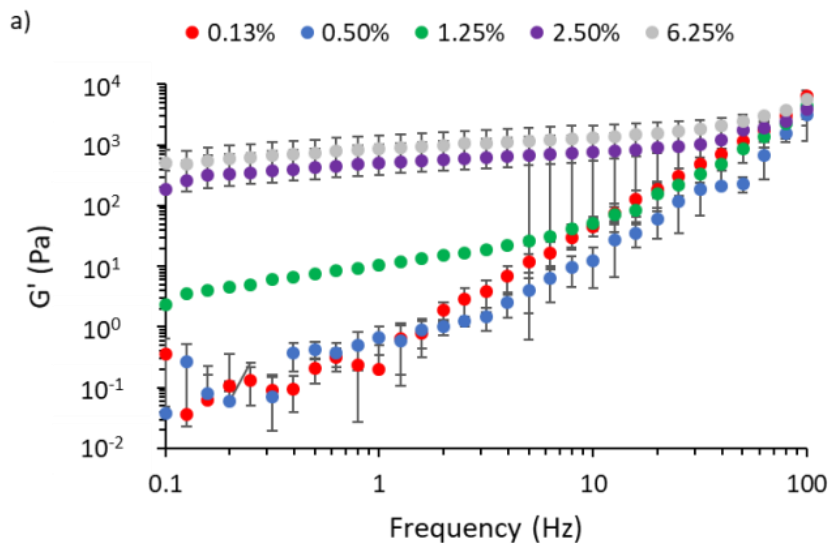

b)

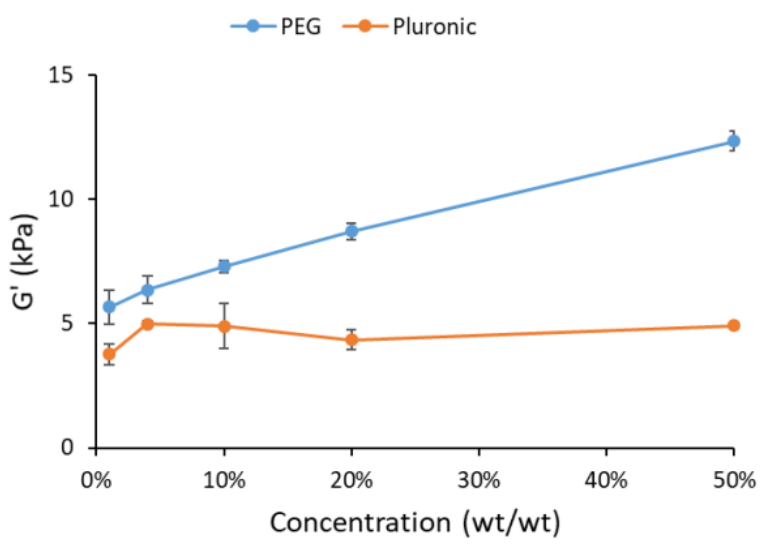

Figure 3. a) Frequency sweeps on PEG gels with weight concentrations from $0.13 \%$ to $6.25 \%$ and $b$ ) comparison of the impact of PEG and Pluronic ${ }^{\circledR}$ on the shear moduli of CMC gels, measured by oscillatory rheology, with wt/wt concentrations of PEG/Pluronic ${ }^{\circledR}$ ranging from $1 \%$ - $50 \%$ compared to the wt concentration of CMC. The concentration of CMC was kept at 12.5 wt\% for all gels. All error bars are standard deviations.

As the PEG concentration increases, the moduli of gels were found to increase, presumably as a result of increased chain entanglement (Figure 3). The frequency sweeps also showed a strong frequency dependency of PEG gels particularly at low PEG concentrations. The addition of PEG to CMC gels increases the moduli of the corresponding materials (Figure $3 b$ ), in a concentration dependent manner. However, this is not seen in the case of Pluronic ${ }^{\circledR}$. This suggests that the PEG is forming an interpenetrating network with the $\mathrm{CMC}$ resulting in an increased modulus while the Pluronic ${ }^{\circledR}$ does not promote intramolecular interactions and entanglement. The 
formation of an interpenetrating network between PEG and CMC may increase the likelihood of competition between the PEG and CMC when cationic salts are added as crosslinkers. ${ }^{40}$

\section{Impact of Inorganic Electrolytes on the Hydrodynamic Diameter of CMC chains}

To gain further insight into the impact of inorganic cations on the molecular structure of CMC hydrogels, we next examined the effect of these electrolytes on changes in hydrodynamic diameter of dilute CMC solutions (therefore focusing on individual polymer chains). We first examined the evolution of the hydrodynamic diameter and viscosity of dilute solutions as a function of CMC concentration (Figure 4). Varying the concentration of CMC solutions from 0.001 to $0.1 \mathrm{wt} \%$, it was observed that the hydrodynamic diameters measured initially increased steadily (below $0.01 \mathrm{wt} \%$ ), before reaching a plateau ${ }^{41}$ at higher concentrations. In parallel, the viscosity of solutions increased monotonically as the polymer concentration increased, even after the hydrodynamic diameter plateau was reached. As the $\mathrm{pH}$ of these solutions was kept constant (therefore resulting in comparable charge densities), this indicates that CMC chains gradually assemble into small aggregates, reaching stable sizes above $0.01 \mathrm{wt} \%$. However, it should be noted that the light scattering set up used, and the $632.8 \mathrm{~nm}$ wavelength of the light source, are not adapted for particles/polymers with hydrodynamic diameters above $500 \mathrm{~nm}$, as secondary scattering events may potentially occur but are ignored by the data processing protocol. ${ }^{41,42}$

Varying the concentration of two inorganic divalent cations, $\mathrm{Ca}^{2+}$ and $\mathrm{Sr}^{2+}$, had a strong impact on the conformation of polymer chains in dilute solutions (based on dynamic light scattering data, see Figure 4b). Hence hydrodynamic diameters decreased rapidly as the concentration of these two cations increased. This was slightly more pronounced in the case of $\mathrm{Ca}^{2+}$. Such a trend implies that CMC chains (and aggregates) are complexed by divalent cations and partially collapse, as a result of intramolecular crosslinking. It could also be argued that divalent cations may contribute to dissociate aggregates seen at higher concentrations, perhaps as they compete with carboxylic moieties and associated hydrogen bonding. It has been shown that polyelectrolytes in the presence of oppositely charged nanocolloids form micelles, where polyelectrolyte chains collapse due to ionic interactions with the oppositely charged nanocolloids. ${ }^{43}$ Similarly increased ionic strength has also been shown to result in a decrease in the hydrodynamic diameter of sodium hyaluronate, ${ }^{44}$ a wormlike charged polysaccharide. Hence our results confirm the strong impact of divalent inorganic cations on CMC chain conformation and intermolecular bonding. This implies that the lack of change in rheological profiles observed in PEG/CMC hydrogels results from the lack of mechanical coupling between densely crosslinked and collapsed nodes, rather than the absence of interactions between divalent cations and CMC molecules. 

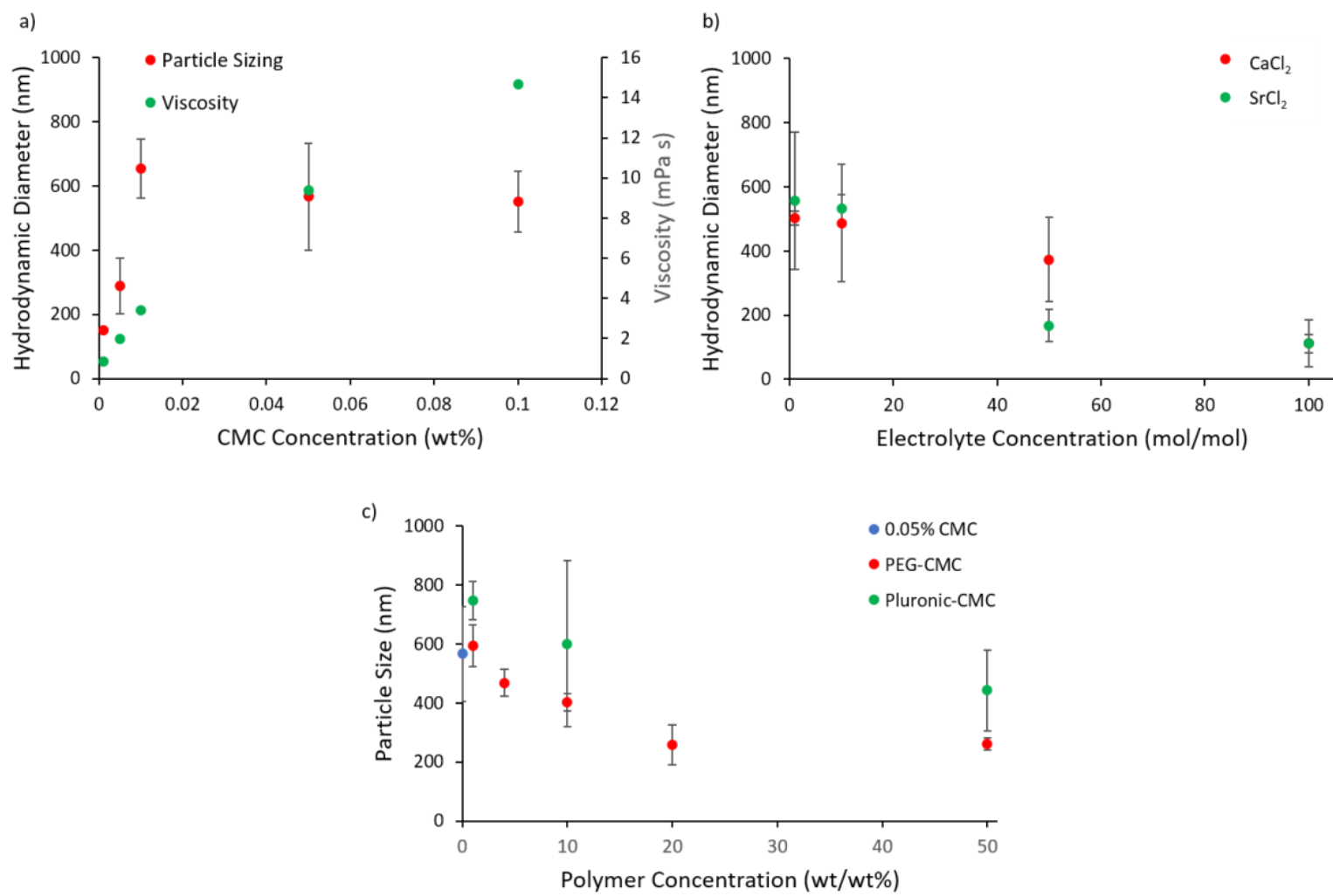

Figure 4. a) Hydrodynamic diameter $\left(D_{H}\right)$ and viscosity measurements of dilute CMC solutions with concentrations ranging from $0.001-0.1 \mathrm{wt} \%$. b) $\mathrm{D}_{\mathrm{H}}$ of particles formed in $0.05 \mathrm{wt} \% \mathrm{CMC}$ solutions with $1 \%$ to $100 \% \mathrm{~mol} / \mathrm{mol}$ inorganic electrolyte relative to the molar concentration of CMC repeat units and c) $D_{H}$ of particles formed in 0.05 wt\% CMC solutions with 1 to $50 \mathrm{wt} / \mathrm{wt} \%$ PEG or Pluronic ${ }^{\circledR}$ relative to the weight concentration of CMC. All error bars are standard deviations.

The influence of PEG and Pluronic ${ }^{\circledR}$ on the hydrodynamic diameter of particles formed in mixed CMC solutions was also investigated. Results indicate that increasing the PEG concentration causes a decrease in the particle size, from $590 \mathrm{~nm}$ at $1 \%$ PEG to $260 \mathrm{~nm}$ at 20\%, plateauing at PEG concentrations above $20 \mathrm{wt} / \mathrm{wt} \%$. Therefore, PEG chains are interacting with $\mathrm{CMC}$ chains and either able to dissociate them and prevent the formation of aggregates, or result in a change of their conformation and partial collapse of their hydrodynamic diameter, similar to what is seen with polysaccharide/protein coacervates. ${ }^{45}$ The interpenetrating network formed by the weakly coupled PEG chains and CMC network does, however, interact across aggregates, as demonstrated by the increase in the moduli of PEG/CMC gels with increasing PEG. Pluronic ${ }^{\circledR}$ induces a less significant decrease in hydrodynamic size, which correlates well with the rheological data obtained for mixed CMC gels. Together, these results suggest weaker interactions between Pluronic ${ }^{\circledR}$ and $\mathrm{CMC}$ chains, compared to PEG/CMC.

Owing to the relatively strong interactions observed between PEG and CMC chains, we next examined the impact of electrolytes on CMC gels in the absence of PEG or Pluronic ${ }^{\circledR}$ chains. In addition, as resulting gels were overall stiffer, they also proved suitable for the characterisation of adhesive properties via lap shear assay, as samples would be sufficiently mechanically stable. 


\section{Impact of Inorganic Electrolytes on CMC Gels}

The CMC concentration and molar ratios of inorganic cations used are shown in Table 2. Results from the oscillatory rheometry, lap shear testing for these conditions are shown in Figure 6.

Table 2. Composition of CMC gel and electrolyte concentrations tested.

\begin{tabular}{llll}
\hline $\begin{array}{l}\text { Sample } \\
\text { Name }\end{array}$ & $\begin{array}{l}\text { CMC } \\
\text { Concentration } \\
\text { /wt\% }\end{array}$ & $\begin{array}{l}\text { lonic } \\
\text { Salt }\end{array}$ & $\begin{array}{l}\text { Molar } \\
\text { Ratio } \\
\text { (CMC:Salt) }\end{array}$ \\
\hline $\mathrm{CaCl}_{2}-20$ & 12.5 & $\mathrm{CaCl}_{2}$ & $20: 1$ \\
$\mathrm{CaCl}_{2}-5$ & 12.5 & $\mathrm{CaCl}_{2}$ & $5: 1$ \\
$\mathrm{CaCl}_{2}-2$ & 12.5 & $\mathrm{CaCl}_{2}$ & $2: 1$ \\
$\mathrm{CaCl}_{2}-1$ & 12.5 & $\mathrm{CaCl}_{2}$ & $1: 1$ \\
$\mathrm{SrCl}_{2}-20$ & 12.5 & $\mathrm{SrCl}_{2}$ & $20: 1$ \\
$\mathrm{SrCl}_{2}-5$ & 12.5 & $\mathrm{SrCl}_{2}$ & $5: 1$ \\
$\mathrm{SrCl}_{2}-2$ & 12.5 & $\mathrm{SrCl}_{2}$ & $2: 1$ \\
$\mathrm{SrCl}_{2}-1$ & 12.5 & $\mathrm{SrCl}_{2}$ & $1: 1$ \\
\hline
\end{tabular}

We found that, at the highest concentrations of electrolyte added, we began to see slight precipitation (Figure 5). This was assumed to be the result of the CMC chains collapsing in the presence of the inorganic divalent cations, resulting in the formation of denser complexes and increasing the heterogeneity of the corresponding hydrogels.

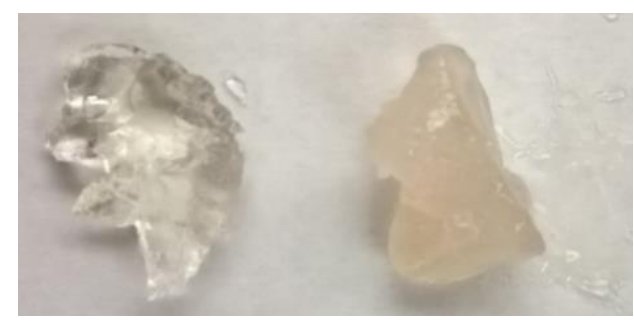

Figure 5. Image of $\mathrm{CMC}$ gels with a low (20:1) $\mathrm{CMC}$ : $\mathrm{CaCl}_{2}$ ratio (left) and a high (1:1) ratio (right). It is clear that at high electrolyte concentrations $\mathrm{CMC}$ gels are more heterogenous and chains start to precipitate, with increased opacity of the corresponding materials.

Precipitation at high electrolyte concentration is also observable in the lap shear profiles, particularly for the $\mathrm{CMC}_{-} \mathrm{CaCl}_{2}$ samples, for which we observed an initial increase in the adhesive shear strength as the electrolyte concentration increased, before a drop at the highest concentrations (Figure 6b). 

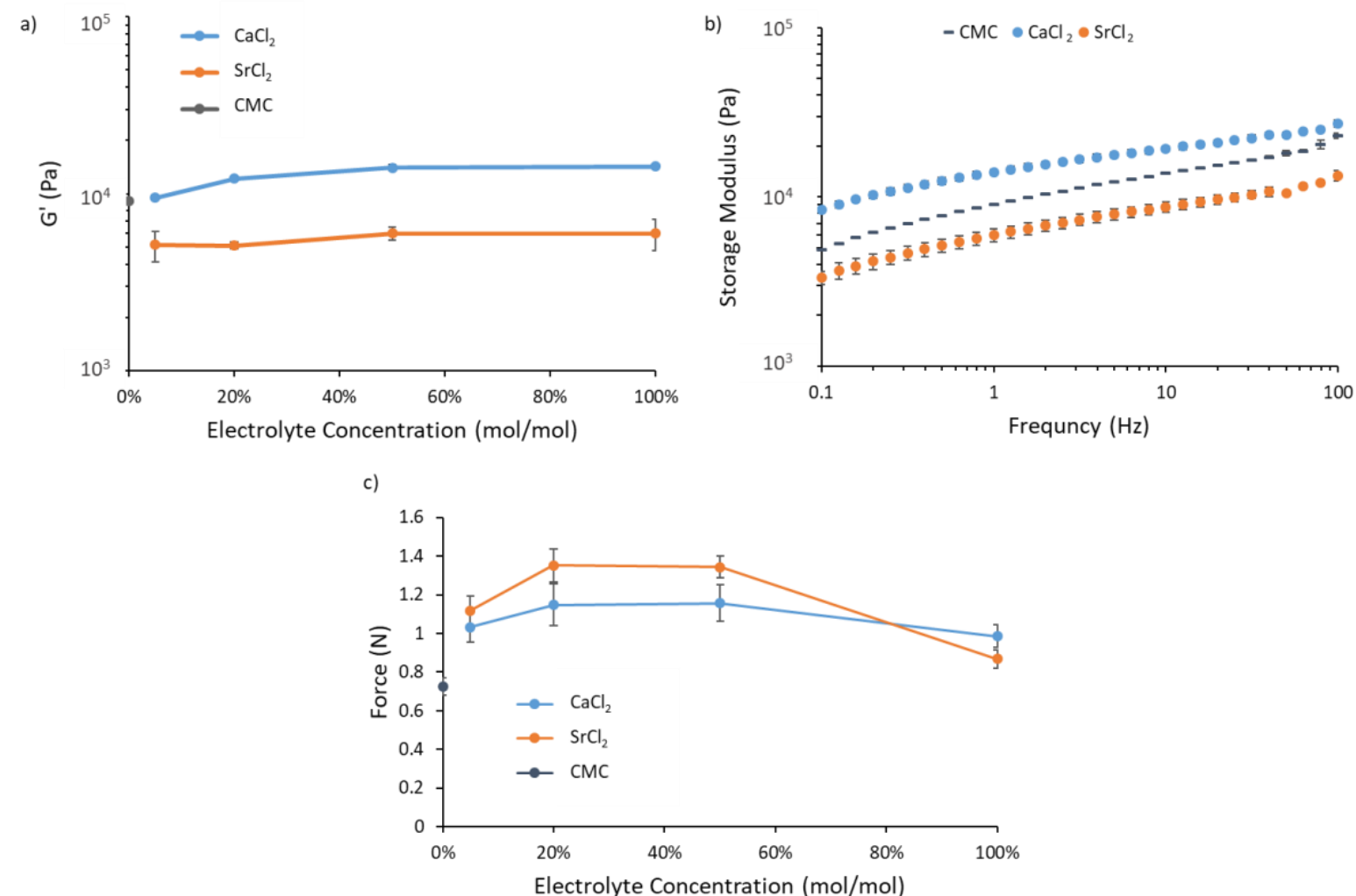

Figure 6. a) Characterisation of CMC gels with varying concentration of inorganic divalent cations by oscillatory rheology. Moduli measured at the different electrolyte concentrations, at $1 \mathrm{~Hz}$ are reported. b) Representative frequency sweeps at an electrolyte concentration of 20\%. c) Summary of the lap shear data showing the peak adhesive force measured for CMC gels as a function of electrolyte concentration (molar concentration with respect to the carboxylic acid moieties in $\mathrm{CMC}$ ). A minimum of three samples per experiment were tested. All error bars are standard deviations.

Figure 6 gathers results from the oscillatory rheology and lap shear testing carried out on CMC gels in the presence of divalent cations $\mathrm{Ca}^{2+}$ and $\mathrm{Sr}^{2+}$ (See Table 2 for details of formulations). From the oscillatory rheology frequency sweeps, it is clear that the shear moduli of CMC gels with and without electrolyte have a strong frequency dependence. This is again expected due to the morphology of the CMC gels, with high hydration, and their physical crosslinking, leading to strong viscoelastic or poro-viscoelastic properties. ${ }^{46,47}$ Furthermore, the increase in moduli with increasing electrolyte concentrations seen with the addition of $\mathrm{CaCl}_{2}$ implies that electrostatic interactions between $\mathrm{CMC}$ chains and $\mathrm{Ca}^{2+}$ cations are strengthening the gel network. This phenomenon has been demonstrated in other similar polyelectrolyte polysaccharides such as alginate ${ }^{38}$ and xanthan. ${ }^{16}$ Ionic crosslinks in CMC solutions have also been shown to increase viscosity, implying some degree of bridging between CMC chains. ${ }^{48}$ Furthermore the strong frequency dependence of the shear modulus of soft physical gels is expected in the presence of ionic crosslinks. ${ }^{46,48}$

The increase in shear moduli with increasing $\mathrm{CaCl}_{2}$ concentration correlates with the lap shear data, with increasing peak force at increasing ionic strength. This is in agreement with the overall cohesive failure of these hydrogels in lap shear assays. However, in the lap shear data, the peak force begins to decrease again at the highest salt concentrations (1:1 molar ratio) whereas shear moduli remain stable at these concentrations. Furthermore, the trend in the rheology data as a function of $\mathrm{CaCl}_{2}$ concentration was not reproduced with the addition of $\mathrm{SrCl}_{2}$, although the lap shear data indicates increased adhesion at intermediate $\mathrm{SrCl}_{2}$ concentrations 
(20-50\%). Similar trends had been observed with the addition of electrolytes to gellan gum, with a significant increase in the ultimate tensile strength measured for associated materials, despite stable Young's moduli. ${ }^{49}$ This could imply that ionic crosslinks increase energy dissipation within associated networks, which increases their ultimate tensile strength, but does not significantly influence Young's moduli.

In agreement with these observations, we had observed that the hydrodynamic diameter of polymer chains and aggregates decreased as a function of electrolyte concentration (Figure 4b). This implies that, in the presence of divalent cations, CMC chains and aggregates are collapsing rather than forming bridges between chains, as would be expected at high dilutions. Increases in shear moduli observed at higher $\mathrm{Ca}^{2+}$ concentrations may indicate that these electrolytes are able to bridge macromolecules and clusters of macromolecules, contributing to the mechanical properties of the macroscopy network. However, the larger radius and polarizability of $\mathrm{Sr}^{2+}$ results in weaker interactions with CMC molecules (Figure 6) and, in turn, weaker associated changes in hydrodynamic diameters and shear moduli, perhaps even shielding interactions between carboxylic groups. In contrast, in lap shear tests, $\mathrm{Sr}^{2+}$ ions may promote softer microstructures and may limit the propagation of dislocations and fractures, allowing the delay of sample failure.

Overall, our results indicate that the addition of divalent cations does not only generate crosslinks between CMC chains, but also results in chain collapse and the formation of isolated "hard" clusters that do not contribute mechanically to the macroscopic rheological properties of the materials and balance intramolecular interactions and electrostatic crosslinking. Hence, two opposing effects impact on CMC hydrogels macroscale mechanics. Therefore, we proposed that polymeric crosslinkers that display terminal cationic "handles" would allow simultaneous complexation of CMC chains, as well as acting as tethers between the resulting hard cores, to strengthen hydrogel mechanics.

\section{Impact of Organic Cationic Crosslinkers on CMC Hydrogel Mechanics}

In order to examine how multivalent organic cationic species would impact the mechanical properties of CMC hydrogels, we selected diethylene triamine (DETA), pentaethylene hexamine (PEHA) and two triblock copolymers of PEI-PEG-PEI differing in the length of their cationic "handles" (Figure 1). The formulation of associated hydrogels, the type of crosslinkers used and their concentrations are presented in Table 3. To induce the protonation of these molecules the $\mathrm{pH}$ of the mixtures used was lowered using $\mathrm{HCl}$ at molar ratios of 3:1 and 6:1, for DETA and PEHA respectively. These results were compared to formulations generated at neutral $\mathrm{pH}$.

To synthesise these block copolymers, we used a precursor route based on the synthesis of poly(2-ethyl-2oxazoline) block copolymers that can be hydrolysed in acidic conditions, resulting in well-controlled linear block copolymer architectures, as presented in Figure 1. Two block copolymers were selected, with a degree of polymerisation of the PEG block of 71 and 10 and 35 ethylene imine repeat units, respectively. 
Table 3. Composition of $\mathrm{CMC}$ gels and organic crosslinker concentrations tested

\begin{tabular}{|c|c|c|c|}
\hline Sample Name & $\begin{array}{l}\mathrm{CMC} \\
\text { Concentration } \\
\text { /wt\% }\end{array}$ & $\begin{array}{l}\text { Ionic } \\
\text { Salt }\end{array}$ & $\begin{array}{l}\text { Molar Ratio (CMC: } \\
\text { X-Linker: } \mathrm{HCL} \text { ) }\end{array}$ \\
\hline bloc 10 - 20 & 12.5 & bloc 10 & $20: 1: 0$ \\
\hline bloc 10 - 5 & 12.5 & bloc 10 & $5: 1: 0$ \\
\hline bloc 10 - 2 & 12.5 & bloc 10 & $2: 1: 0$ \\
\hline bloc 10 - 1 & 12.5 & bloc 10 & $1: 1: 0$ \\
\hline bloc 35 - 20 & 12.5 & bloc 35 & 20:1:0 \\
\hline bloc 35 - 5 & 12.5 & bloc 35 & $5: 1: 0$ \\
\hline bloc 35 - 2 & 12.5 & bloc 35 & $2: 1: 0$ \\
\hline bloc 35 - 1 & 12.5 & bloc 35 & $1: 1: 0$ \\
\hline DETA - 20 & 12.5 & DETA & $20: 1: 0$ \\
\hline DETA - 5 & 12.5 & DETA & $5: 1: 0$ \\
\hline DETA - 2 & 12.5 & DETA & $2: 1: 0$ \\
\hline DETA - 1 & 12.5 & DETA & $1: 1: 0$ \\
\hline PEHA - 20 & 12.5 & PEHA & 20:1:0 \\
\hline PEHA - 5 & 12.5 & PEHA & 5:1:0 \\
\hline PEHA - 2 & 12.5 & PEHA & $2: 1: 0$ \\
\hline PEHA - 1 & 12.5 & PEHA & 1:1:0 \\
\hline DETA HI - 20 & 12.5 & DETA & $20: 1: 3$ \\
\hline DETA HCl - 5 & 12.5 & DETA & $5: 1: 3$ \\
\hline DETA HCl - 2 & 12.5 & DETA & $2: 1: 3$ \\
\hline DETA HCl - 1 & 12.5 & DETA & $1: 1: 3$ \\
\hline PEHA HCl - 20 & 12.5 & PEHA & $20: 1: 6$ \\
\hline PEHA HCl - 5 & 12.5 & PEHA & $5: 1: 6$ \\
\hline PEHA HCl - 2 & 12.5 & PEHA & $2: 1: 6$ \\
\hline PEHA HCl - 1 & 12.5 & PEHA & 1:1:6 \\
\hline
\end{tabular}


a)

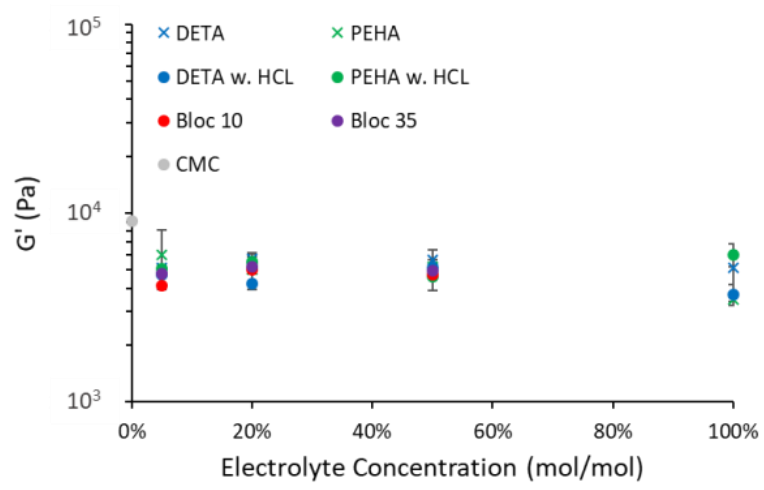

c)

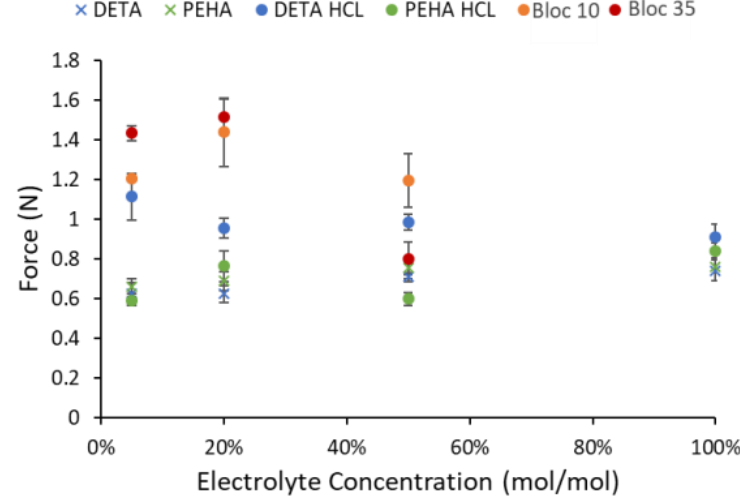

b) - CMC $\times$ DETA $\times$ PEHA $\bullet$ DETA HCL $\bullet$ PEHA HCL $\bullet$ Bloc $10 \bullet$ Bloc 35
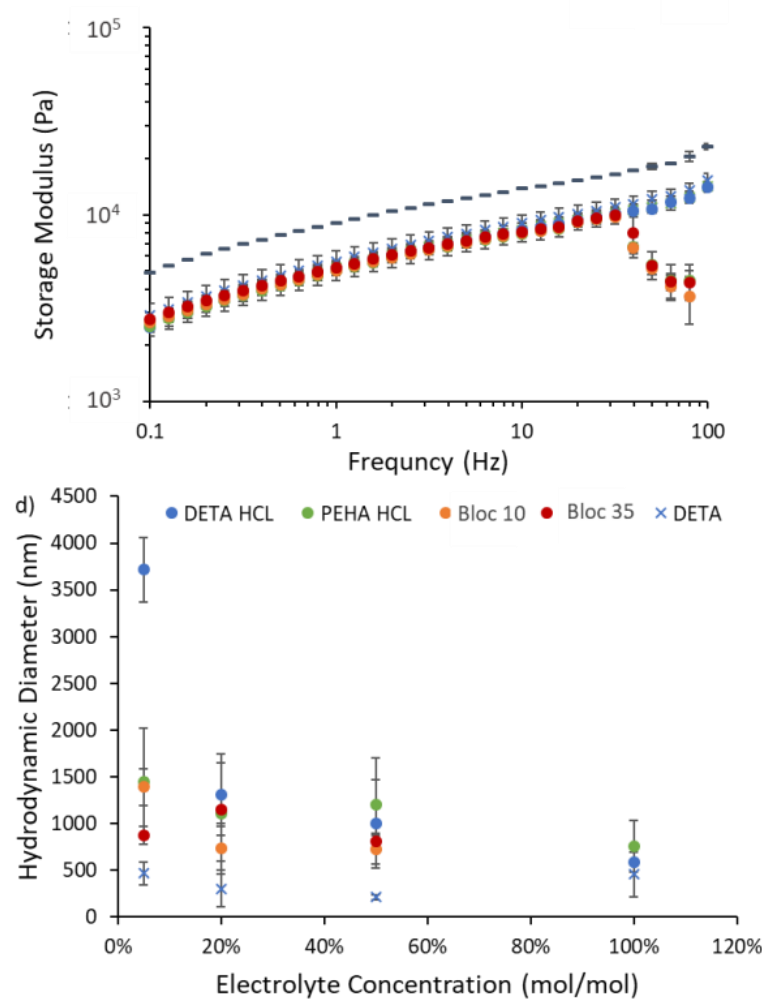

Figure 7. Characterisation of $\mathrm{CMC}$ gels with varying concentrations of organic cations (where the $\mathrm{mol} / \mathrm{mol} \mathrm{ratio}$ is the ratio of amines per carboxylic acid) by oscillatory rheology (a and b). a) Summary of shear moduli measured at different electrolyte concentrations at $1 \mathrm{~Hz}$. b) Representative frequency sweeps at an organic electrolyte concentration of $20 \%$. c) Lap shear data plotted as the peak adhesive force measured for CMC gels at varying organic binders molar concentrations. d) Light scattering data obtained for CMC gels in the presence of varying organic electrolyte concentrations, with a CMC weight concentration of $0.05 \%$. The ratio of $\mathrm{CMC} /$ organic cation was kept constant for all characterisation. A minimum of three samples per experiment were tested. All error bars are standard deviations.

The shear modulus of CMC gels with and without the organic crosslinkers was strongly frequency dependent (Figure 7), in agreement with expected physical crosslinking and results obtained for inorganic electrolytes. However, there is no significant increase in shear moduli with any of the organic binders studied. Similarly, polysaccharide based coacervates such as BSA/pectin, ${ }^{45} \mathrm{BSA} / \mathrm{k}$-carrageenan, ${ }^{50}$ gelatin/sodium montmorillonite ${ }^{51}$ and O-carboxymethyl chitosan/gum Arabic ${ }^{52}$ show similar frequency dependent rheological properties arising from physical crosslinking of corresponding hydrogels. Furthermore increasing the ionic strength of the solution resulted in a modest decrease in the shear moduli of many of these gels, ${ }^{45,50}$ without significant matrix strengthening.

In contrast, we observed a clear increase in the peak adhesive strength, up to $1.6 \mathrm{~N}$, in lap shear tests (Figure 7c). This is particularly clear for the block copolymers, for which a sharp increase is seen at low concentrations. This effect was more pronounced for longer PEI blocks at lower crosslinker concentrations. PEHA and DETA without protonation, display little to no impact on the adhesive shear strength, implying that electrostatic interactions play an important role in adhesive behaviour and the energy dissipation during fracture. As was the case for the inorganic electrolytes, all samples tested by lap shear failed cohesively with clear sample residues still covering both sides of the lap shear testing geometries. 
Dynamic light scattering was used next, to explore molecular interactions between the different organic cations and CMC chains. Significantly larger particle sizes resulted from the addition of the block copolymers, when compared to the inorganic salts (apart from the addition of unprotonated DETA), with particle sizes in the range of 550-3000 nm in the presence of organic cations, compared to 100-550 nm with the inorganic electrolytes. This suggests significant bridging between CMC chains with the addition of such multivalent organic electrolytes. However, at higher cation concentrations, the hydrodynamic diameters of CMC complexes decreased in size, as was observed for inorganic cations. Therefore, our results indicate that, although organic cations are bridging across several $\mathrm{CMC}$ chains and bringing them together to former larger complexes at low mole ratios, they result in the collapse of $\mathrm{CMC}$ aggregates at higher concentrations and mole ratios (as proposed in Figure 8). This phenomenon is seen in other coacervate systems where, if the ionic strength of the solution is not carefully controlled, the electrolytes will complex and precipitate rather than forming a two phase coacervate gel. ${ }^{30,33,34}$ In contrast, unprotonated DETA did not display initial increase in complex size and did not result in CMC chain collapse, in line with reduced charge and the absence of significant hydrogen bonding between the components of this system, in aqueous conditions. Therefore, we propose that the bridging of CMC chains by organic cations results in very little change in the bulk mechanics of CMC gels due to the local collapse of CMC chains. In contrast, the adhesive shear strength of the corresponding gels was significantly improved at low binder concentration, presumably due to stronger fracture healing properties of the associated networks.

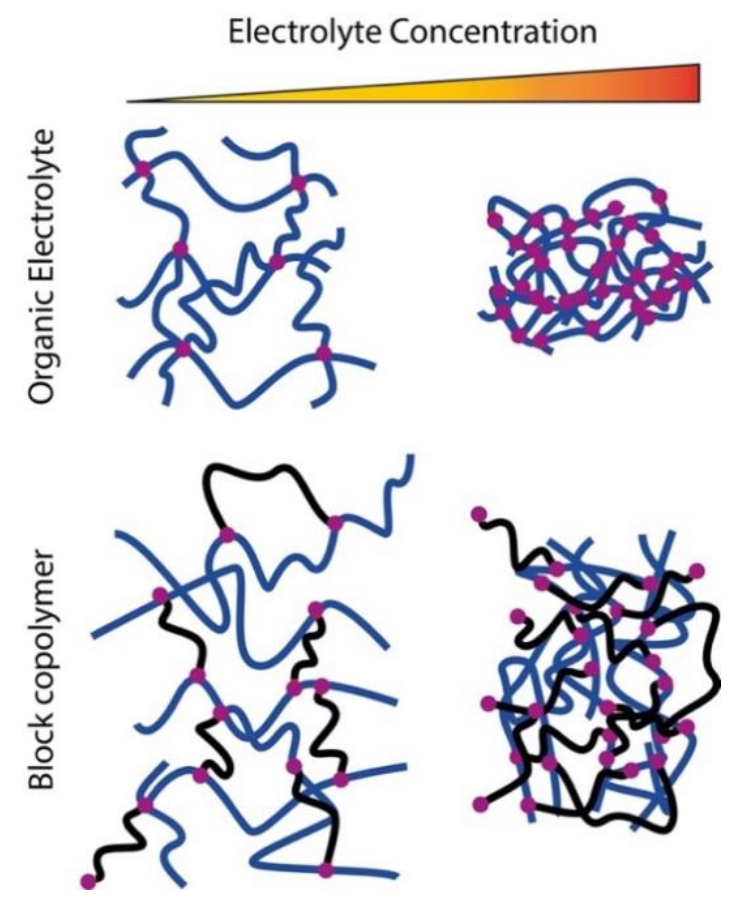

Figure 8. Schematic representation of the proposed impact of organic electrolytes and block copolymer on CMC chain conformation and aggregation. 


\section{Conclusions}

The impact of inorganic divalent cationic crosslinkers and organic cationic polyelectrolyte crosslinkers on CMC gel rheology and adhesive strength was studied. It was found that such electrostatic crosslinking had little impact on rheological properties of resulting hydrogels, but significantly impacted adhesive strength. When the ionic crosslinker concentration was high, gels became cloudy, indicative of precipitation. This behaviour correlates with the hydrodynamic diameter of chains and aggregates studied in dilute solutions, which decreased at increasing electrolyte concentrations. Our data indicates that the addition of inorganic cationic electrolytes results in the complexation and collapse of CMC chains rather than their crosslinking. Similar trends were observed in the case of organic electrolytes, but overlapped with an initial formation of aggregates at low electrolyte concentrations, in particular in the case of block copolymer structures that enable more efficient crosslinking via coacervation. At higher concentrations, these aggregates were observed to partially collapse or reduce in size. Overall, our results indicate that more polarisable inorganic divalent cations $\left(\mathrm{Sr}^{2+}\right)$ and organic oligo(ethylene imines) increase the conformational collapse associated with corresponding complexes more significantly than harder inorganic cations $\left(\mathrm{Ca}^{2+}\right)$. In turn, this is proposed to result in weaker rheological properties (storage shear moduli).

Although intramolecular interactions mediated by electrolytes had a modest impact on shear moduli, they significantly impacted the adhesive strength of the corresponding materials. This suggests that the complexes formed and ionic crosslinks did result in energy dissipation conferring to the gels a greater adhesive strength. Therefore, our results indicate that coacervate hydrogels formed between cationic polyelectrolytes and CMC generate weakly bound phases that do not mechanically stiffen hydrogels but enhance adhesive strength. Further refinement of macromolecular architecture may enable the modulation of such properties over a broader range.

\section{Experimental Section}

General. Calcium chloride (Bioreagent grade), strontium chloride (ACS reagent, 99\%), diethylenetriamine (DETA, reagent plus, 99\%), pentaethylenehexamine (PEHA, technical grade), PEG-di-p-tosylate (average $M_{n} 3,500$ ), 2ethyl-2-oxazoline, F127 pluronic ${ }^{\circledR}$ (bioreagent grade), poly(ethylene glycol) (reagent grade) and hydrochloric acid $(\mathrm{HCl})$ were all purchased from Sigma Aldrich and used as received, apart from 2-ethyl-2-oxazoline, which was freshly distilled directly prior to polymerisation. Sodium carboxymethyl cellulose (CMC) (250,000 Mw) was purchased from Ashland Chemicals.

PEI-PEG-PEI block copolymer synthesis

Triblock copolymers of poly(ethylene imine)-b-poly(ethylene glycol)-b-poly(ethylene imine) (PEI-PEG-PEI) were synthesised according to the following protocols. A poly(2-ethyl-2-oxazoline)- $b$-poly(ethylene glycol)- $b$-poly(2ethyl-2-oxazoline) (PEtOx-PEG-PEtOx) (targeted degrees of polymerisations for the three blocks of 10:71:10 and 35:71:35) was first produced. For a 10:80:10 block copolymer, $0.205 \mathrm{~g}$ bis-tosylate-terminated poly(ethylene glycol) $\left(\mathrm{Mn}_{\mathrm{n}}, 3,500 \mathrm{~g} / \mathrm{mol} ; 0.07 \mathrm{mmol}\right), 0.142 \mathrm{~g}$ 2-ethyl-2-oxazoline $(1.42 \mathrm{mmol}$ ) and $2 \mathrm{~mL}$ anhydrous acetonitrile were added to a thoroughly dried (oven) microwave vial. The vial was placed in the microwave synthesizer (CEM, Discover) and the reaction solution was heated to $160^{\circ} \mathrm{C}$, over $30 \mathrm{~s}$, and held for $10 \mathrm{~min}$ at this temperature. The vial was then cooled to room temperature under gas flow. The reaction solution was next heated to $80^{\circ} \mathrm{C}$ for $12 \mathrm{~h}$. After the acetonitrile was evaporated the polymer was dissolved in chloroform. The solution was 
extracted three times in a saturated sodium bicarbonate aqueous solution, followed by three extractions in brine. Finally, the solution was dried with potassium sulfate and filtered before evaporating the solvent under vacuum. The PEtOx-PEG-PEtOx (35:71:35) was synthesised using the same protocol, however 0.497 g of 2-ethyl2-oxazoline was used. This afforded light yellow solids (yield: 10:71:10, 63\%; 35:71:35, 53\%). SEC (DMF). PEtOxPEG-PEtOx (10:71:10), Mn, 9,970 g/mol, Mw 13,970 g/mol. ${ }^{1} \mathrm{H}$ NMR (400 MHz, D $\left.{ }_{2} \mathrm{O}\right) . \delta$ (ppm), 3.5-3.7 (m, $\left.\mathrm{OCH}_{2} \mathrm{CH}_{2}\right), 3.2-3.6\left(\mathrm{~m}, \mathrm{NCH}_{2} \mathrm{CH}_{2}\right)$, 2.1-2.5 (m, $\left.\mathrm{COCH}_{2}\right), 1.0-1.1\left(\mathrm{~m}, \mathrm{CCH}_{3}\right) . \mathrm{FTIR} . v\left(\mathrm{~cm}^{-1}\right), 2875$ (C-H stretch), 1718 ( $\mathrm{C}=\mathrm{O}$ stretch), $1630(\mathrm{C}=\mathrm{O}), 1100(\mathrm{C}-\mathrm{H})$. The PEtOx-PEG-PEtOx block copolymers were then hydrolysed by dissolving $0.4 \mathrm{~g}(0.1 \mathrm{mmol})$ in $12 \mathrm{~mL} 0.5 \mathrm{M} \mathrm{HCl}$ in a one neck round bottom flask and placing under reflux at $120^{\circ} \mathrm{C}$ overnight under inert nitrogen atmosphere. Finally, the acid solution was removed under reduced pressure. This afforded a white solid (yield: 95\%). ${ }^{1} \mathrm{H}$ NMR (400 MHz, $\left.\mathrm{D}_{2} \mathrm{O}\right): \delta(\mathrm{ppm}), 3.5-3.7\left(\mathrm{~m}, \mathrm{OCH}_{2} \mathrm{CH}_{2}\right), 3.1-$ $4.1\left(\mathrm{~m}, \mathrm{NHCH}_{2} \mathrm{CH}_{2}\right)$; note residual peaks associated with incomplete hydrolysis, especially for longer PEtOx block copolymers (22\% residual). FTIR: $v\left(\mathrm{~cm}^{-1}\right), 3450$ ( $\mathrm{N}-\mathrm{H}$ stretch), 2983 (C-H stretch next to amine), 2860 (C-H stretch), 1654 (N-H bend), 1420 (C-H bend), 1160 (C-N stretch).

Hydrogel sample preparation

CMC was mixed with deionised water and the desired electrolyte solutions (see Tables 1-3 for details of compositions). The $\mathrm{CMC}$ and the electrolyte solutions were prepared separately and once both were completely homogenous, they were mixed together. If electrolytes were added as neat to the dissolved CMC, they resulted $^{22}$ in localised precipitation and a relatively heterogeneous mixture, so ensuring the $\mathrm{CMC}$ and salt solutions were completely homogenous prior to their combination proved essential. To control the $\mathrm{pH}$, hydrochloric acid was added to the electrolyte solution prior to mixing with the CMC solution. For samples introducing PEG within the formulation, preliminary tests focused on CMC and PEG concentrations of 3 and $1.5 \%$, respectively. These preliminary samples were characterised via oscillatory rheology only. As the viscosity of these samples was found to be too low for adhesive formulations, later characterisation focused on gels at 12.5 wt\% CMC concentrations. Once the CMC and electrolyte solutions were mixed and appeared homogenous they were centrifuged at $4000 \mathrm{rpm}$ for $2 \mathrm{~min}$ to remove bubbles. This process was repeated until a clear homogenous sample was obtained. For the preparation of PEG and CMC mixed solutions, these two polymers were mixed together as dry powders before dissolution at the desired concentration in deionised water and subsequent mixing with the appropriate electrolyte solution.

Dynamic light scattering and electrophoretic light scattering measurements

Samples prepared for light scattering measurements were significantly more dilute than for hydrogel formulation, but were mixed according to the same protocol. Hydrodynamic diameters were characterised at identical ratios of CMC to cationic crosslinker as for corresponding hydrogels (see Tables 2 and 3), but the concentration of CMC was only 0.05 wt\%. Dynamic light scattering measurements were carried out using a Nano-ZS Zetasizer from Malvern. $1 \mathrm{~mL}$ of sample solution was added to a $2.5 \mathrm{~mL}$ VWR PS macro Cuvette. Prior to measurements, a 1 min dwell time was allowed once the sample was loaded. Scans were repeated 3 times per sample with 13 scans per repeat at a wavelength of $632.8 \mathrm{~nm}$ and a back scattering angle of $173^{\circ}$. A minimum of three samples were tested for each set of conditions. Data analysis was performed using the Malvern Zetasizer software 7.02 and the hydrodynamic particle size determined using the Stokes-Einstein equation. ${ }^{35}$ Nanoparticles were assumed to be spherical, monodisperse and non-interacting.

Oscillatory Rheology

Oscillatory rheology was performed using a Discovery Hybrid Rheometer 3 (DHR3) from TA Instruments. Characterisation was performed using a steel $20 \mathrm{~mm}$ parallel plate top geometry and a steel Peltier plate as the bottom geometry. Samples were characterised by applying a frequency sweep first, with an oscillating displacement of $10^{-4} \mathrm{rad}$ and a frequency range of $0.1-100 \mathrm{~Hz}$. This was followed by an amplitude sweep at an 
oscillating frequency of $1 \mathrm{~Hz}$ from $10^{-5}$ to $10^{-3} \mathrm{rad}$. All assays were performed at room temperature and a $30 \mathrm{~s}$ dwell time was allowed between protocols. A minimum of three repeats on each sample was carried out.

Lap Shear Testing

Lap shear testing was performed using an Instron 5943 Universal Testing System with a $500 \mathrm{~N}$ load cell. $2 \mathrm{~g}$ of material were placed at one end of a PMMA slide. The gel was then covered by an overlapping slide, with an overlap area of 25 by $25 \mathrm{~mm}$. The sample was then compressed between the two slides with a force of $90 \mathrm{~N}$ for $5 \mathrm{~s}$. The load was then removed and the sample was left to relax for $5 \mathrm{~min}$ before being loaded with $90 \mathrm{~N}$ force again for $5 \mathrm{~s}$. The sample was then mounted and the test started as quickly as possible. The sample was deformed at a rate of $50 \mathrm{~mm} / \mathrm{min}$ until failure. A minimum of 5 repeats per sample were performed.

Instrumentation for Polymer Chemistry Characterisation

${ }^{1}$ H NMR spectroscopy was carried out using a Bruker AVIII 400. ATR-FTIR spectra were produced using a Bruker Tensor 27 spectrometer equipped with a MCT detector. Results were acquired at a resolution of $16 \mathrm{~cm}^{-1}$ and a total of 128 scans per run in the region of $600-4000 \mathrm{~cm}^{-1}$. GPC analysis was performed using an Agilent 1260 Infinity system equipped with a refractive index and variable wavelength detector, 2 PLgel $5 \mu \mathrm{m}$ mixed-C column $(300 \times 7.5 \mathrm{~mm})$, and a PLgel $5 \mathrm{~mm}$ guard column $(50 \times 7.5 \mathrm{~mm})$ operated in DMF with $\mathrm{NH}_{4} \mathrm{BF}_{4}(5 \mathrm{mM})$. The instrument was calibrated with poly(methyl methacrylate) standards (5.5 to $46.9 \mathrm{~kg} / \mathrm{mol}$ ). All samples were filtered through a $0.2 \mu$ m nylon-66 membrane before analysis.

\section{Acknowledgements}

Funding from the Engineering and Physical Sciences Research Council (EP/L505602/1) and the China Scholarship Council (201306890005) are gratefully acknowledged.

\section{Supplementary Material}

Details of FTIR and NMR characterisation of the synthesised block copolymers of poly(ethylene imine) and poly(ethyene glycol) are presented in the Supplementary Material file associated with this manuscript.

\section{References}

1. Clasen, C.; Kulicke, W. M. Prog. Polym. Sci. 2001, 26 (9), 1839-1919.

2. Chang, C. Y.; Zhang, L. N. Carbohydrate Polym. 2011, 84 (1), 40-53.

3. Klemm, D.; Heublein, B.; Fink, H. P.; Bohn, A. Angew. Chemie. Int. Ed. 2005, 44 (22), 3358-3393.

4. Drury, J. L.; Mooney, D. J., Biomaterials 2003, 24 (24), 4337-4351.

5. Lionetto, F.; Sannino, A.; Mensitieri, G.; Maffezzoli, A., Macromol. Symp., Wiley Online Library: 2003; pp 199-208.

6. Desmarais, A. J., Denture adhesive composition. US Patent 5,024,701 (1991), to Hercules Inc.

7. Kulak, Y.; Özcan, M.; Arikan, A., J. Prosthodont, 2005, 14 (4), 248-252.

8. Han, J. M.; Hong, G.; Dilinuer, M.; Lin, H.; Zheng, G.; Wang, X. Z.; Sasaki, K., Acta Odontol. Scand. 2014, $72(8), 839-845$.

9. An, Y. R.; Li, D. Y.; Roohpour, N.; Gautrot, J. E.; Barber, A. H., Dent. Mater. 2016, 32 (5), 615-623. 
10. de Oliveira, N. M.; Rodriguez, L. S.; Marin, D. O. M.; Paleari, A. G.; Pero, A. C.; Compagnoni, M. A., J. Prosthet. Dent. 2014, 112 (5), 1182-1187.

11. Papadiochou, S.; Emmanouil, L.; Papadiochos, L., J. Prosthet. Dent. 2015, 113 (5), 391-397.

12. Adinugraha, M. P.; Marseno, D. W.; Haryadi, Carbohydrate Polym. 2005, 62 (2), 164-169.

13. Benchabane, A.; Bekkour, K., Colloid Polym. Sci. 2008, 286 (10), 1173-1180.

14. Heinze, T.; Koschella, A., Macromol. Symp. 2005, 223 (1), 13-40.

15. Drury, J. L.; Dennis, R. G.; Mooney, D. J., Biomaterials 2004, 25 (16), 3187-3199.

16. Choppe, E.; Puaud, F.; Nicolai, T.; Benyahia, L., Carbohydrate Polym. 2010, 82 (4), 1228-1235.

17. Nickerson, M. T.; Paulson, A. T.; Speers, R. A., Food Hydrocoll. 2003, 17 (5), 577-583.

18. Augst, A. D.; Kong, H. J.; Mooney, D. J., Macromol. Biosci. 2006, 6 (8), 623-633.

19. Lee, K. Y.; Rowley, J. A.; Eiselt, P.; Moy, E. M.; Bouhadir, K. H.; Mooney, D. J., Macromolecules 2000, 33 (11), 4291-4294.

20. Lee, K. Y.; Mooney, D. J., Prog. Polym. Sci. 2012, 37 (1), 106-126.

21. Schmitt, C.; Turgeon, S. L., Adv. Colloid Interface Sci. 2011, 167 (1), 63-70.

22. Weinbreck, F.; Tromp, R. H.; de Kruif, C. G., Biomacromolecules 2004, 5 (4), 1437-1445.

23. Shang, J.; Shao, Z.; Chen, X., Biomacromolecules 2008, 9 (4), 1208-1213.

24. Zhao, Q.; Qian, J.; An, Q.; Gao, C.; Gui, Z.; Jin, H., J. Membr. Sci. 2009, 333 (1), 68-78.

25. Zhivkov, A. M., Cellulose-Fundamental Aspects, InTech: 2013.

26. Feng, X.; Pelton, R.; Leduc, M., Ind. Eng. Chem. Res. 2006, 45 (20), 6665-6671.

27. Ye, A. J., Int. J. Food Sci. Technol. 2008, 43 (3), 406-415.

28. Turgeon, S. L.; Schmitt, C.; Sanchez, C., Curr. Opin. Colloid Interface Sci. 2007, 12 (4), 166-178.

29. Wang, Y.; Kimura, K.; Huang, Q.; Dubin, P. L.; Jaeger, W., Macromolecules 1999, 32 (21), 7128-7134.

30. Krogstad, D. V.; Choi, S. H.; Lynd, N. A.; Audus, D. J.; Perry, S. L.; Gopez, J. D.; Hawker, C. J.; Kramer, E. J.; Tirrell, M. V., J. Phys. Chem. B 2014, 118 (45), 13011-13018.

31. Priftis, D.; Farina, R.; Tirrell, M., Langmuir 2012, 28 (23), 8721-8729.

32. Krogstad, D. V.; Lynd, N. A.; Choi, S. H.; Spruell, J. M.; Hawker, C. J.; Kramer, E. J.; Tirrell, M. V., Macromolecules 2013, 46 (4), 1512-1518.

33. Chollakup, R.; Smitthipong, W.; Eisenbach, C. D.; Tirrell, M., Macromolecules 2010, 43 (5), 2518-2528.

34. Wang, Q.; Schlenoff, J. B., Macromolecules 2014, 47 (9), 3108-3116.

35. Attard, P.; Antelmi, D.; Larson, I., Langmuir 2000, 16 (4), 1542-1552.

36. Tang, Y.-F.; Du, Y.-M.; Hu, X.-W.; Shi, X.-W.; Kennedy, J. F., Carbohydrate Polym. 2007, 67 (4), 491-499.

37. Ramazani-Harandi, M. J.; Zohuriaan-Mehr, M. J.; Yousefi, A. A.; Ershad-Langroudi, A.; Kabiri, K., Polym.

Test. 2006, 25 (4), 470-474.

38. Kuo, C. K.; Ma, P. X., Biomaterials 2001, 22 (6), 511-521.

39. Silva-Correia, J.; Oliveira, J. M.; Caridade, S.; Oliveira, J. T.; Sousa, R.; Mano, J.; Reis, R. J., J. Tissue Eng.

Regen. Med. 2011, 5 (6), e97-e107.

40. Steed, J. W., Coord. Chem. Rev. 2001, 215 (1), 171-221.

41. Key, P.; Sellen, D., J. Polym. Sci. Polym. Phys. 1982, 20 (4), 659-679.

42. Sellen, D., J. Polym. Sci. B Polym. Phys. 1987, 25 (4), 699-716.

43. Qi, L.; Chapel, J.-P.; Castaing, J.-C.; Fresnais, J.; Berret, J.-F., Soft Matter 2008, 4 (3), 577-585.

44. Fouissac, E.; Milas, M.; Rinaudo, M.; Borsali, R., Macromolecules 1992, 25 (21), 5613-5617.

45. Ru, Q.; Wang, Y.; Lee, J.; Ding, Y.; Huang, Q., Carbohydrate Polym. 2012, 88 (3), 838-846.

46. Galli, M.; Comley, K. S. C.; Shean, T. A. V.; Oyen, M. L., J. Mater. Res. 2009, 24 (3), 973-979.

47. Zhao, X.; Huebsch, N.; Mooney, D. J.; Suo, Z., J. App. Phys. 2010, 107 (6). 
48. Matsumoto, T.; Mashiko, K. J. P. E.; Science 1988, 28 (6), 393-402.

49. Coutinho, D. F.; Sant, S. V.; Shin, H.; Oliveira, J. T.; Gomes, M. E.; Neves, N. M.; Khademhosseini, A.; Reis, R. L., Biomaterials 2010, 31 (29), 7494-7502.

50. Chai, C.; Lee, J.; Huang, Q., LWT - Food Sci. Technol. 2014, 59 (1), 356-360.

51. Qazvini, N. T.; Bolisetty, S.; Adamcik, J.; Mezzenga, R., Biomacromolecules 2012, 13 (7), 2136-2147.

52. Huang, G. Q.; Xiao, J. X.; Wang, S. Q.; Qiu, H. W., Food Hydrocoll. 2015, 43, 436-441.

This paper is an open access article distributed under the terms of the Creative Commons Attribution (CC BY) license (http://creativecommons.org/licenses/by/4.0/) 